\title{
Évaluation et analyse de sensibilité du modèle CERES-Maize en conditions alsaciennes
}

\author{
S Plantureux 1, P Girardin 2, D Fouquet 1, JY Chapot 2 \\ 1 INRA-ENSAIA, laboratoire agronomie et environnement, 2, av de la Forêt-de-Haye, 54500 Vandœuvre-lès-Nancy; \\ 2 INRA, station d'agronomie, 28, rue de Herlisheim, BP 507, 68021 Colmar Cedex, France
}

(Reçu le 25 août 1989; accepté le 28 octobre 1990)

\begin{abstract}
Résumé - CERES-Maize est un modèle de simulation de la croissance et du développement du maïs élaboré et validé aux Etats-Unis. Afin d'estimer les possibilités de transposition du modèle dans des conditions européennes, des simulations ont été réalisées pour 2 variétés de maïs (LG11 et DEA) cultivées en Alsace 2 années consécutives. Pour chaque variété, le modèle a été calibré sur 1 année et validé sur la suivante. L'analyse de sensibilité des paramètres liés à la variété et au sol montre que la réponse du modèle varie fortement selon la nature du paramètre et les conditions agro-climatiques considérées dans l'analyse. En outre, les paramètres du modèle sont nombreux et non indépendants, ce qui rend le calibrage délicat sur le plan mathématique. D'un point de vue méthodologique, l'analyse de sensibilité de modèles tels que CERES-Maize et la généralisation du calibrage peuvent donc poser d'importants problèmes.

Dans les conditions agro-climatiques des simulations (Alsace, sol limoneux, années 1978, 1979, 1986 et 1987), le modèle prédit correctement le développement et la croissance du maïs. L'ajustement de paramètres est alors nécessaire, mais la structure du modèle n'est pas remise en cause. Une généralisation à l'ensemble de l'Europe nécessiterait par contre la modification du modèle, en particulier pour la partie qui simule la phase d'implantation de la culture.
\end{abstract}

CERES-Maize $/$ modélisation $/$ Zea mays = maïs / calibrage $/$ validation $/$ sensibilité

Summary - Evaluation and sensitivity analysis of the CERES-Maize model in North-Eastern France. CERESMaize is a simulation model of maize growth and development, conceived and successfully used in the United States. In this study, CERES-Maize was tested under European conditions, then adapted to these conditions. Two field experiments were carried out for 2 years in North-Eastern France (Alsace) using 2 cultivars (LG11 and DEA) on a loamy soil. Yearly nitrogen fertilization was $150 \mathrm{~kg}^{\cdot h a^{-1}}(N)$. No irrigation was performed. Calibration and validation of the model were conducted for each cultivar, followed by sensitivity analysis of the newly calibrated model for the LG11 variety. Genetic constants, ie growing degree days (base $8^{\circ} \mathrm{C}$ ) from seedling emergence to the end of the juvenile phase $\left(P_{1}\right)$, potential kernel number $\left(\mathrm{G}_{2}\right)$ and potential kernel growth rate $\left(\mathrm{G}_{3}\right)$, are generally unknown for hybrids grown in Europe (table I). Their values were therefore estimated from data given for early hybrids grown in Southern Canada and from French experiments testing DEA and LG11 (table II).

Results showed that predictions given by the non-calibrated model using estimated constants are generally very different from measured values (table III). Therefore, genetic constants and equations calculating leaf area were modified (fig 2). Then, validations taken from other data sets than those used for calibration showed a good agreement between observed and predicted values for plant development and grain yield (tables $I V$ and VI). Growth variables are still slightly underestimated by the model. This might be due to the fact that parameters used for calculating conversion of radiation in dry matter and repartition of dry matter between organs are not correct as regards European conditions. The sensitivity analysis revealed a great sensivity of genetic and hydraulic parameters (figs 3, 5 and 6). The sensitivity depends on simulation conditions, especially on the occurrence of water or nitrogen stress (fig 3). Other soil characteristic parameters were not found to be as sensitive as albedo (fig 4).

CERES-Maize can be used for simulation of maize growth, development and for grain yield prediction in European conditions. However, a thorough calibration of the model and further experiments to accurately determine parameters unknown in Europe (eg end of the juvenile phase) are required. Furthermore, some subroutines should be included to take into account specific conditions such as cold springs and hydromorphic soils.

CERES-Maize $/$ modelling $/$ Zea mays = maize $/$ parameter ranking $/$ validation $/$ sensitivity analysis 


\section{INTRODUCTION}

Les objectifs de la modélisation de la croissance et du développement des plantes de grande culture sont multiples (Whisler et al, 1986) et parfois contradictoires :

- synthétiser un ensemble de connaissances sur le fonctionnement d'une plante ou d'un peuplement et mettre en évidence les domaines où cette connaissance est insuffisante en établissant une hiérarchie parmi les problèmes à résoudre (aide à la compréhension).

- prévoir l'influence du climat, des techniques culturales et de leurs interactions sur le comportement du végétal et

- en déduire des informations utiles aux praticiens, aux agents économiques, aux décideurs politiques et aux chercheurs (outil d'aide à la décision),

- simuler de nombreuses situations afin de mieux raisonner les expérimentations à mettre en place (aide à l'expérimentation).

Face à ces objectifs, des modèles de type mécaniste ou plus rarement stochastique ont été développés. Si la comparaison de ces approches n'est pas le propos du présent article, notons que chaque type de modèle ne peut a priori répondre à l'ensemble des objectifs décrits ci-dessus. D'un point de vue théorique, les modèles mécanistes présentent l'avantage d'être explicatifs et de s'adapter à des contextes variés.

Cherchant à développer un modèle mécaniste capable de simuler la croissance et le développement du maïs dans nos conditions, nous nous sommes d'abord interrogés sur la possibilité de transposer des modèles élaborés dans d'autres conditions. Parmi ceux-ci, le modèle CERESMaize nous est apparu comme le plus complet et le plus achevé.

Elaboré aux Etats-Unis par des équipes d'agronomes, de pédologues et de physiologistes de I'USDA, CERES-Maize (Jones et al, 1984) peut être considéré comme un modèle de type mécaniste, bien qu'une partie du fonctionnement de la plante soit modélisée à partir de relations purement statistiques. Dans son contexte agro-climatique, la simulation par le modèle apparaît comme réaliste (Berti et al, 1986). CERES-Maize a même été utilisé avec succès pour simuler les effets de défoliations dues à la grêle (Baanante et al, 1986; Piper et Weiss, 1988 ) et également pour la prévision du rende- ment en grain à l'échelle régionale dans le Cornbelt (Hodges et al, 1987). Cette dernière utilisation peut d'ailleurs apparaître, du point de vue théorique, comme une aberration pour un modèle conçu à l'échelle de la parcelle. Ce changement d'échelle entraîne des simplifications et/ou des généralisations discutables.

Quel que soit l'objectif pour lequel le modèle CERES-Maize pourrait être utilisé, une étude préalable du comportement du modèle dans les conditions françaises s'avère indispensable (tests de sensibilité des paramètres, évaluation).

Dans cette optique, nous avons testé le modèle CERES-Maize à partir de résultats d'essais réalisés en Alsace. Ce travail a d'abord consisté à évaluer le modèle dans sa configuration native, puis à estimer l'importance des modifications nécessaires à l'adaptation du modèle aux conditions européennes : simple ajustement de paramètres ou remise en cause de la structure de tout ou partie du modèle, et donc de ses hypothèses de départ.

\section{MATÉRIEL ET MÉTHODES}

La validation du modèle CERES-Maize dans les conditions alsaciennes a été effectuée à partir d'essais réalisés en 1978 et 1979 (variété LG11) et en 1986 et 1987 (variété DEA). Les densités de semis sont respectivement de 75000 plantes/ha pour LG11 et pour DEA de 100000 plantes/ha. Les 2 variétés ont été cultivées sans irrigation, avec une fertilisation azotée de 150 unités/ha. Aucun stress hydrique ou azoté important n'a pu être noté au champ. Toutes les expérimentations ont été menées sur un sol de type limoneux profond.

\section{Description du modèle CERES-Maize}

Le modèle CERES-Maize est un modèle de simulation de la croissance et du développement du maïs. Les principes détaillés de son fonctionnement ont été décrits antérieurement (Jones et Kiniry, 1986) et nous ne reviendrons que sur les principales caractéristiques du modèle (fig 1).

CERES-Maize est proposé en 2 versions : "standard" et "azote". La version "standard" considère la nutrition de la plante comme non limitante et ignore l'effet des agents pathogènes et de la verse mécanique. La version "azote" permet de tenir compte d'éventuels stress azotés. Le programme, écrit en FORTRAN, commence par une phase d'initialisation (lecture des données d'entrée, mise à 0 de compteurs de degrés-jours) puis calcule à un pas de temps journalier : 


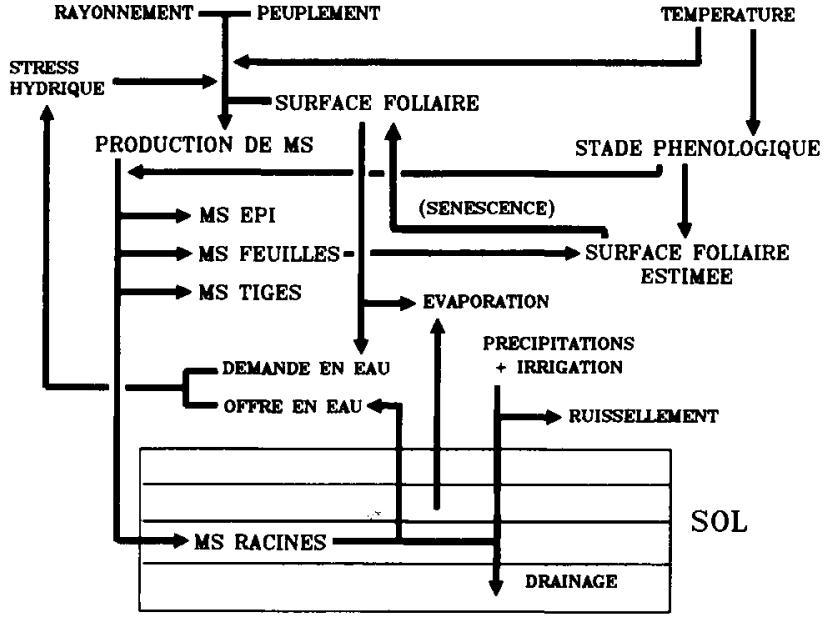

Fig 1. Représentation schématique du fonctionnement du modèle CERES-Maize (version "standard»).

- le bilan hydrique pour les différents horizons de sol que l'on peut distinguer dans le profil (sousprogramme WATBAL),

- le déroulement de la phénologie (sous-programme PHENOL),

- la croissance en matière sèche (MS) des organes de la plante (racines, tige, feuilles, grains, etc) et la surface foliaire (sous-programme GROSUB),

- le bilan azoté (minéralisation, dénitrification, lessivage, absorption racinaire) par horizon de sol (sousprogrammes MINIMO, DNIT, NFLUX et NUPTAKE), pour la version "azote».

En outre, le modèle détermine les principales composantes du rendement (nombre de grains par $\mathrm{m}^{2}$ ou par épi, poids d'un grain). II estime aussi, pour différentes phases du développement, des coefficients de stress hydrique qui interviennent dans le calcul de l'accroissement journalier de la biomasse totale et de la surface foliaire, ainsi que dans la répartition de la matière sèche entre les feuilles, les tiges et l'épi.

Les informations nécessaires au fonctionnement du modèle concernent le climat, le sol, les techniques culturales et la variété (tableau I). Les conditions initiales d'humidité du sol et éventuellement de teneur en azote organique et minéral (version "azote») doivent être précisées. La simulation peut débuter avant le jour du semis, le programme ne calculant alors que le bilan hydrique du sol.

\section{RÉSULTATS ET DISCUSSION}

\section{Evaluation de CERES-Maize}

L'évaluation de CERES-Maize a consisté à calibrer le modèle pour une année et à valider ce calibrage l'année suivante. Un tel traitement a concerné la variété LG11 en 1978 et 1979, et la variété DEA en 1986 et 1987.
Tableau I. Données d'entrée du modèle CERESMaize.

- Précipitations, rayonnement global, températures minimales et maximales

Sol : 1) par horizon (au maximum 10 horizons)

- épaisseur de chaque horizon

- caractéristiques hydriques (point de flétrissement permanent, capacité au champ, saturation)

- coefficient d'enracinement

2) Pour l'ensemble du sol

- albédo du sol nu

- limite d'évaporation potentielle

- conductivité hydraulique

- coefficient de ruissellement

\section{Techniques culturales}

- semis : date, profondeur, densité

- irrigation : dates et doses

Variété : Paramètres génétiques

$P_{1}=$ Somme des degrés-jour (base $8^{\circ} \mathrm{C}$ ) entre levée et fin phase juvénile

$P_{2}=$ Coefficient de sensibilité à la photopériode

$P_{5}=$ Somme des degrés-jour (base $8^{\circ} \mathrm{C}$ ) entre floraison femelle et maturité physiologique

$G_{2}=$ Nombre potentiel de grains par plante

$\mathrm{G}_{3}=$ Taux potentiel de croissance du grain en $\mathrm{mg} /$ degré-jour

Le choix des paramètres de calibrage s'est effectué en 2 temps :

Une analyse de sensibilité a d'abord été effectuée avec l'ensemble des paramètres du modèle pour les classer en fonction de leur sensibilité. Ce classement a été établi en considérant la variation relative du rendement et de la variable de sortie la plus directement influencée par le paramètre en question (par exemple, la date de floraison pour le paramètre $P_{1}$ ). Nous ne présentons pas cette analyse dont les conclusions rejoignent celles de l'analyse réalisée après calibrage présentée ci-dessous.

Parmi les paramètres les plus sensibles du modèle, nous n'avons ensuite retenu pour le calibrage que les paramètres génétiques. Le nombre important de paramètres de CERESMaize et les possibilités actuelles du calcul numérique empêchent pratiquement d'envisager un calibrage du modèle sur tous les paramètres. Nous avons donc choisi, parmi les paramètres les plus sensibles du modèle, ceux dont la mesure ou l'estimation nous semblaient la plus déli- 
Tableau II. Valeur des paramètres génétiques avant calibrage du modèle.

LG11

$P_{1}$ (levée - fin phase juvénile) $\left(^{\circ} \mathrm{J}\right)$

$P_{2}$ (sensibilité photopériodique)

$P_{5}$ (floraison - maturité physiologique) $\left({ }^{\circ} \mathrm{J}\right)$

$\mathrm{G}_{2}$ (nombre potentiel de grains par plante)

$\mathrm{G}_{3}$ (Taux potentiel de croissance du grain) $\mathrm{mg} \cdot \mathrm{j}^{-1} \cdot \mathrm{plante}^{-1}$

$\begin{array}{cc}125 & 180 \\ 0 & 0 \\ 600 & 740 \\ 600 & 600 \\ 7,0 & 7,0\end{array}$

cate : les paramètres liés à la variété. Ainsi, la somme de température (base $8^{\circ} \mathrm{C}$ ) nécessaire pour aller de l'émergence à la fin de la phase juvénile n'est connue pour aucune variété utilisée en Europe.

Le modèle a d'abord été calibré pour chaque variété (LG11 et DEA). Le calibrage a été effectué en faisant varier systématiquement et parfois simultanément les paramètres, afin d'ajuster d'abord la phénologie puis la croissance. Nous avons alors procédé à une évaluation en comparant les valeurs observées et simulées du rendement en grain, de ses composantes, ainsi que de la surface foliaire.

Les valeurs des paramètres génétiques, inconnues en France pour les variétés LG11 et DEA, ont été d'abord estimées à partir d'informations concernant des hybrides américains de précocité comparable, et de valeurs calculées dans nos conditions pour ces 2 variétés (Bloc et al, 1983; Gay et Bloc, 1983) (tableau II).

Dans ces conditions, la simulation s'écarte sensiblement de la réalité, sur le plan de la phénologie et surtout de la croissance des différentes parties de la plante (tableau III). On remarque, en particulier, une sous-estimation importante de l'indice foliaire ( $L A I)$ qui se traduit par un rendement en grain et en biomasse aérienne inférieurs aux mesures réalisées au champ.

Compte tenu de l'écart important entre les indices foliaires ( $L A I)$ mesurés et calculés, l'indice foliaire a fait l'objet d'un calibrage (fig 2). La sous-estimation par le modèle provient en particulier de la différence du nombre de feuilles entre les variétés européennes et les variétés américaines qui ont servi à calibrer cette équation. De plus, la surface foliaire toujours verte est mal prédite lors de la phase de sénescence. Remarquons que cette estimation est délicate sur le terrain, ce qui peut expliquer de légères différences entre les observations réalisées en France et aux Etats-Unis.
Le modèle ainsi calibré pour l'année 1978 (tableau IV) a fait l'objet d'une validation pour 1979 (tableau V). On note alors que la prévision de la phénologie, de la surface foliaire et du rendement restent corrects en conservant pour 1979 les paramètres calibrés pour 1978. En outre la valeur de ces paramètres est tout à fait réaliste.

La même démarche a été menée en calibrant le modèle en 1986 pour la variété DEA et en validant en 1987 (tableau VI). Dans ce cas, le calibrage a porté sur les paramètres génétiques, les modifications précédentes dans les équations qui permettent de calculer l'indice foliaire (LAI) étant conservées. Dans cette situation, le modèle présente le même comportement que pour LG11, avec une bonne prévision du rendement et de la phénologie. Notons cependant des écarts importants dans la prévision de la biomasse totale.

D'une manière générale, la phénologie du maïs, qui est étroitement reliée aux sommes de température, est mieux simulée par les modèles que la croissance. En ce qui concerne CERESMaize, on note que la prédiction de la date de floraison est meilleure que celle de la maturité,

Tableau III. Simulation avant calibrage du modèle. Variété LG11, Année 1978, Colmar.

Mesuré Calculé

\begin{tabular}{|c|c|c|}
\hline Date de floraison & 202 & 201 \\
\hline Date de maturité & 277 & 260 \\
\hline Rendement grain $15 \%\left(10^{2} \mathrm{~kg}^{2} \mathrm{ha}^{-1}\right)$ & 106 & 74 \\
\hline Poids de 1000 grains $(\mathrm{g})$ & 313 & 203 \\
\hline Nombre de grains $/ \mathrm{m}^{2}$ & 2813 & 3092 \\
\hline Nombre de grains par épi & 375 & 412 \\
\hline$L A \mid$ à la floraison $\left(\mathrm{m}^{2} / \mathrm{m}^{2}\right)$ & 3,2 & 1,45 \\
\hline Biomasse aérienne en (t/ha) & 18,7 & 12,7 \\
\hline Poids tiges / feuilles en (t/ha) & 9,7 & 6,4 \\
\hline
\end{tabular}

$P_{1}=125 ; P_{2}=0 ; P_{5}=600 ; G_{2}=600 ; G_{3} \doteq 7,0$ 


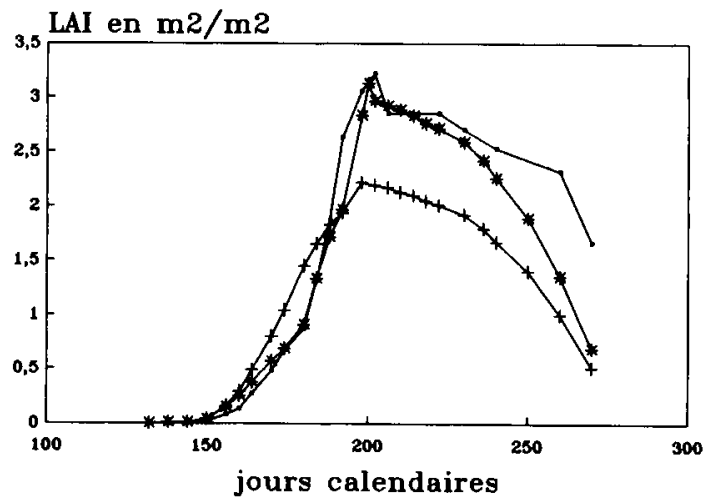

Fig 2. CERES-Maize. Calibrage de l'indice foliaire $L A$; (.) observations Colmar LG11 1978; (+) modèle CERES avant calibrage: PLAG: 3,5 $\times T I \times S W D F_{2} \times X N^{2}$; (") modèle CERES après calibrage: $P L A G=11,5 \times T I \times S W D F_{2} \times$ $X N^{1}, 25 . P L A G=$ accroissement foliaire; $T I=$ degré d'avancement dans un stade phénologique; $S W D F_{2}=$ coefficient de stress hydrique; $X N=$ nombre de feuilles.

difficile à observer au champ. Les résultats de terrain ne mentionnent généralement pas la date de maturité physiologique, mais celle de la récolte, qui est conditionnée par des facteurs pédoclimatiques comme la portance du sol.

Le travail réalisé sur 4 années en un seul lieu ne permet pas de tester statistiquement la qualité prédictive du modèle. Une telle démarche nécessiterait de disposer de données pluri-annuelles très complètes obtenues sur des sites variés pour les comparer aux valeurs simulées. Dans cette optique, il est en effet nécessaire d'avoir un échantillon constitué de nombreuses situations indépendantes (Wallach et Goffinet, 1987).

Tableau IV. Simulation après calibrage du modèle. Variété LG11; année 1978, Colmar.

Mesuré Calculé

\begin{tabular}{lrc}
\hline & & \\
Date de floraison & 202 & 201 \\
Date de maturité & 277 & 278 \\
Rendement grain $15 \%\left(10^{2} \mathrm{~kg} \cdot \mathrm{ha}^{-1}\right)$ & 106 & 107 \\
Poids de 1000 grains (g) & 313 & 321 \\
Nombre de grains/m² & 2813 & 2803 \\
Nombre de grains par épi & 375 & 374 \\
LAlà la floraison (m $\left.2 / \mathrm{m}^{2}\right)$ & 3,2 & 3,13 \\
Biomasse aérienne en (t/ha) & 18,7 & 17,5 \\
Poids tiges / feuilles en (t/ha) & 9,7 & 8,5
\end{tabular}

$P_{1}=125 ; P_{2}=0 ; P_{5}=720 ; G_{2}=500 ; G_{3}=7,2$
Tableau V. Validation du modèle (calibrage en 1978). Variété LG11, année 1979, Colmar.

Mesuré Calculé

\begin{tabular}{lcc} 
Date de floraison & 206 & 197 \\
Date de maturité & 284 & 279 \\
Rendement grain $15 \%\left(10^{2} \mathrm{~kg}^{-} \mathrm{ha}^{-1}\right)$ & 100 & 102 \\
Poids de 1000 grains $(\mathrm{g})$ & - & 308 \\
Nombre de grains/m & - & 2795 \\
Nombre de grains par épi & - & 377 \\
LAlà la floraison $\left(\mathrm{m}^{2} / \mathrm{m}^{2}\right)$ & - & 3,08 \\
Biomasse aérienne en (t/ha) & 18,6 & 17,3 \\
Poids tiges / feuilles en (t/ha) & 10,2 & 8,7 \\
\hline
\end{tabular}

$P_{1}=125 ; P_{2}=0 ; P_{5}=720 ; G_{2}=500 ; G_{3}=7,2$

\section{Analyse de la sensibilité des paramètres}

En modélisation, les tests de sensibilité ont 2 objectifs : ils permettent d'abord de connaître les paramètres qu'il est intéressant de faire varier en vue d'un calibrage du modèle.

Une fois ce modèle calibré, les tests de sensibilité permettent d'estimer la précision nécessaire pour déterminer les paramètres (Jorgensen, 1988). C'est dans cette optique qu'ont été réalisés les tests de sensibilité présentés cidessous.

Les paramètres nécessaires au fonctionnement de CERES-Maize sont nombreux et parfois délicats à estimer. II s'agit des paramètres d'entrée (tableau I) auxquels s'ajoutent les para-

Tableau VI. Validation du modèle (calibrage en 1986) variété DEA, année 1987, Colmar.

Mesuré Calculé

$\begin{array}{lcc}\text { Date de floraison } & 205 & 206 \\ \text { Date de maturité } & 323 & 312 \\ \text { Rendement grain } 15 \%\left(10^{2} \mathrm{~kg}^{-} \mathrm{ha}^{-1}\right) & 126 & 126 \\ \text { Poids de } 1000 \text { grains }(\mathrm{g}) & - & 320 \\ \text { Nombre de grains/m } & - & 3204 \\ \text { Nombre de grains par épi } & - & 320 \\ \text { LAlà la floraison }\left(\mathrm{m}^{2} / \mathrm{m}^{2}\right) & - & 5,2 \\ \text { Biomasse aérienne en (t/ha) } & 20,0 & 23,4 \\ \text { Poids tiges / feuilles en (t/ha) } & 11,0 & 12,8\end{array}$

$P_{1}=125 ; P_{2}=0 ; P_{5}=720 ; G_{2}=500 ; G_{3}=7,2$ 
mètres et constantes des équations internes du modèle.

L'analyse de sensibilité a été réalisée sur l'ensemble des paramètres d'entrée du modèle liés au sol et à la variété. Une telle approche pose cependant plusieurs problèmes d'ordre méthodologique liés aux conditions de réalisation des tests, au domaine de variation et à l'indépendance des paramètres testés.

\section{Importance des conditions de réalisation des tests de sensibilité}

Une première série de tests a d'abord montré que la sensibilité des paramètres est fortement liée aux conditions d'alimentation hydrique et azotée de la plante. Ainsi, l'effet d'une variation de plus ou moins $50 \%$ du paramètre DUL (capacité au champ) sur le rendement a été testé en provoquant ou non un stress hydrique (par limitation des précipitations) et un stress azoté (par suppression de la fertilisation azotée) (fig 3). La diminution de la capacité au champ envisagée dans le cas présent, qui revient en fait à limiter la réserve utile du sol, correspond à une gamme de variation observable au champ.

Dans les situations de stress hydrique $\left(\mathrm{N}+\mathrm{H}_{2} \mathrm{O}\right.$ - et $\left.\mathrm{N}-\mathrm{H}_{2} \mathrm{O}-\right)$ le paramètre $\mathrm{DUL}$ apparaît évidemment plus sensible qu'en l'absence d'un tel stress $\left(\mathrm{N}+\mathrm{H}_{2} \mathrm{O}+\right.$ et $\left.\mathrm{N}-\mathrm{H}_{2} \mathrm{O}+\right)$. Les différences de sensibilité observées dans le cas d'un stress hydrique entre une alimentation normale $\left(\mathrm{N}+\mathrm{H}_{2} \mathrm{O}-\right)$ ou déficiente en azote $\left(\mathrm{N}-\mathrm{H}_{2} \mathrm{O}-\right)$ montrent la complexité de la réponse du modèle à la variation d'un paramètre : ainsi, dans le cas où l'azote n'est pas limitant, le rendement final est fortement affecté. En effet, l'azote entraîne dans ce cas une croissance plus rapide de la plante qui assèche plus vite le sol. Les stress hydriques lors de la floraison et de la phase de remplissage du grain sont de ce fait plus marqués.

Cet exemple, confirmé par de nombreux autres tests, souligne les dangers d'extrapolation des analyses de sensibilité. II conduit donc à considérer avec prudence les conclusions que l'on peut tirer de l'apparente insensibilité de certains paramètres d'un modèle dans des conditions climatiques ou de culture particulières.

\section{Domaine de variation et indépendance des paramètres}

L'analyse de la sensibilité d'un paramètre n'a de sens que si les valeurs du paramètre qui sont testées restent réalistes.
Ainsi le paramètre $\mathrm{G}_{2}$ (nombre de grains potentiel par plante) ne peut varier, pour l'ensemble des variétés cultivées, que de 500 à 900 . Cependant, pour une variété donnée, cette gamme de variation se restreint.

D'autre part, le fait de faire varier $G_{2}$ sur toute la gamme des valeurs possibles implique pratiquement de changer de variété, et donc de modifier d'autres paramètres comme $P_{5}$ (somme de degrés-jour floraison femelle - maturité physiologique). Ces 2 paramètres ne sont en effet pas indépendants.

\section{Analyse de sensibilité}

Conditions : variété LG11, Colmar 1978, avec ou sans stress hydrique et/ou azoté.

\section{Paramètres peu sensibles}

- l'albédo du sol (fig 4), la limite d'évaporation potentielle du sol, la conductivité hydraulique et le coefficient de ruissellement. Ces caractéristiques du sol peuvent n'avoir d'influence sur le rendement final que dans des conditions très particulières. Tel est par exemple le cas du ruissellement qui suppose la conjugaison d'une pluviométrie et d'une humidité de la surface du sol importantes,

- le $\mathrm{pH}$. Ce paramètre intervient dans la minéralisation de l'azote. Cette absence de réaction correspond à un manque de sensibilité du sousmodèle azote.

\section{Paramètres sensibles}

- les paramètres qui déterminent la réserve utile du sol et donc les possibilités d'alimentation hy-

$\%$ du rendement en grain

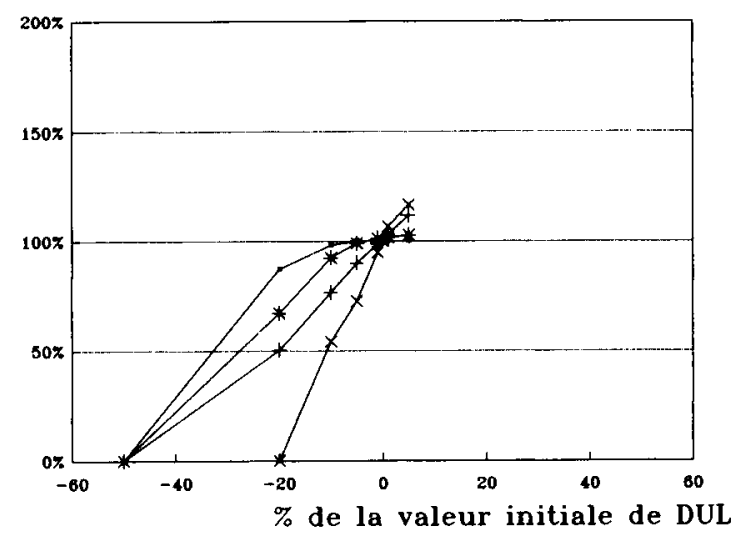

Fig 3. CERES-Maize. Test de sensibilité du paramètre DUL (capacité au champ). (.) absence de stress $=\mathrm{N}(+) \mathrm{H}_{2} \mathrm{O}(+) ;\left({ }^{*}\right)$ stress azoté $=\mathrm{N}(-) \mathrm{H}_{2} \mathrm{O}(+) ;(x)$ stress hydrique $=\mathrm{N}(+) \mathrm{H}_{2} \mathrm{O}(-)$; $(+)$ stress hydrique et azoté $=\mathrm{N}(-) \mathrm{H}_{2} \mathrm{O}(-)$. Une variation de $10 \%$ de $D U L=3 \%$ d'humidité. 
drique de la plante : capacité au champ (DUL) (fig 3 ) et point de flétrissement ( $L L$ ) (fig 5). Ces paramètres doivent donc être déterminés avec précision, une erreur de 5\% (soit 1-2 points d'humidité) entraînant, dans les situations envisagées, une variation de rendement en grain comprise entre 0 et $25 \%$. Généralement ce type de paramètre est obtenu par mesure au laboratoire avec une précision de l'ordre de 5\%;

- les paramètres liés à la variété : $P_{1}, P_{5}$ (fig 6 et 7), $G_{2}, G_{3}$. En fait, il faut considérer avec prudence l'analyse de sensibilité de ces paramètres qui ne sont pas indépendants les uns des autres. Sous cette réserve, on observe que l'augmentation de $G_{2}$ et $G_{3}$ qui sont des valeurs potentielles reliées aux composantes du rendement, accroît proportionnellement la matière sèche du grain jusqu'à un palier. Un autre facteur limitant, le rayonnement, se manifeste alors. Quelles que soient les conditions de stress, le rendement est directement lié à la durée de la phase de remplissage du grain, ce qui explique la sensibilité de $P_{5}$.

La variation de $P_{1}$ avance ou retarde la floraison et décale donc, par rapport au climat, les périodes de sensibilité au stress hydrique ou les périodes à fort rayonnement. Ceci explique les variations plus irrégulières du rendement en fonction de $P_{1}$

Ces exemples de tests montrent que l'on doit déterminer certains paramètres (exemple: DUL, capacité au champ) avec une grande précision. Compte tenu de la difficulté de mesurer correctement la valeur de paramètres tels que $\mathrm{G}_{3}$ (taux de croissance potentiel du grain), il a fallu en ob-

$\%$ du rendement en grain

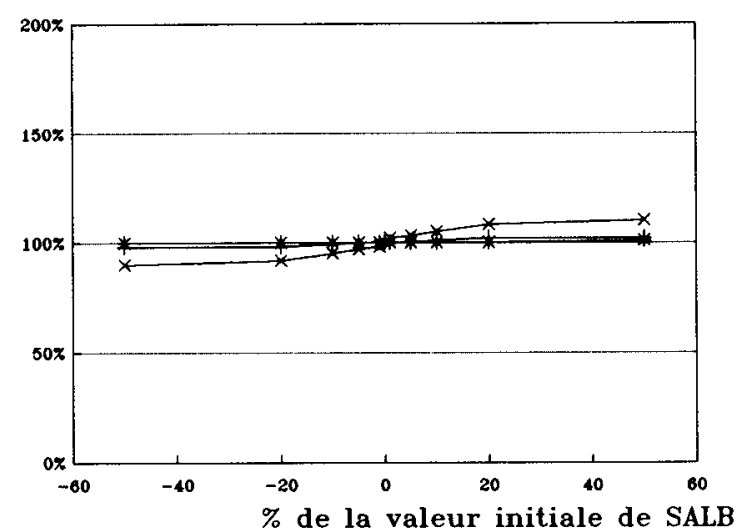

Fig 4. CERES-Maize. Test de sensibilité du paramètre SALB (albedo du sol). (.) absence de stress $=\mathrm{N}(+) \mathrm{H}_{2} \mathrm{O}(+)$; (") stress azoté $=\mathrm{N}(-) \mathrm{H}_{2} \mathrm{O}(+) ;(x)$ stress hydrique $=\mathrm{N}(+) \mathrm{H}_{2} \mathrm{O}(-)$; $(+)$ stress hydrique et azoté $=\mathrm{N}(-) \mathrm{H}_{2} \mathrm{O}(-)$. Une variation de $10 \%$ de $S A L B=0,015$ d'albédo.
$\%$ du rendement en grain

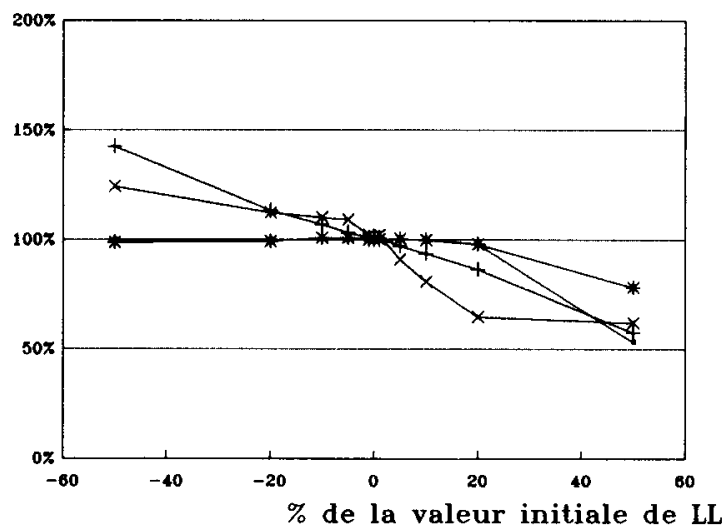

Fig 5. CERES-Maize. Test de sensibilité du paramètre $L L$ (point de flétrissement). (.) absence de stress $=\mathrm{N}(+) \mathrm{H}_{2} \mathrm{O}(+)$; $\left({ }^{*}\right)$ stress azoté $=\mathrm{N}(-) \mathrm{H}_{2} \mathrm{O}(+) ;(x)$ stress hydrique $=\mathrm{N}$ $(+) \mathrm{H}_{2} \mathrm{O}(-) ;(+)$ stress hydrique et azoté $=\mathrm{N}(-) \mathrm{H}_{2} \mathrm{O}(-)$. Une variation de $10 \%$ de $L L=3 \%$ d'humidité.

tenir une estimation par calibrage. II n'en demeure pas moins qu'une mesure réelle est plus souhaitable.

\section{CONCLUSION}

Les simulations réalisées en 1978-1979 et 19861987 montrent que CERES-Maize peut prévoir correctement, dans les conditions alsaciennes et pour ces années, le développement et la croissance d'une culture de maïs. Les données de terrain disponibles actuellement ne permettent cependant pas de calibrer très précisément le modèle dans nos conditions, l'information étant généralement incomplète (suivi de biomasse à

\section{\% du rendement en grain}

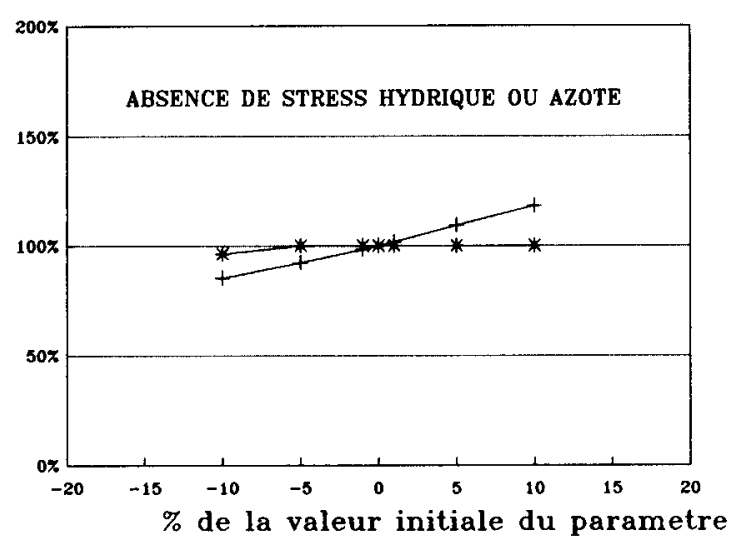

Fig 6. CERES-Maize. Test de sensibilité des paramètres $P_{1}$ et $P_{5}$ en absence de stress hydrique ou azoté. ( $\left.{ }^{*}\right) P_{1}$, somme de degrés-jour entre la levée et la fin de la phase juvénile. (+) $P_{5}$, somme de degrés-jour entre la floraison et la maturité physiologique. Valeurs initiales $(100 \%): P_{1}=125$; $P_{5}=720$. 
\% du rendement en grain

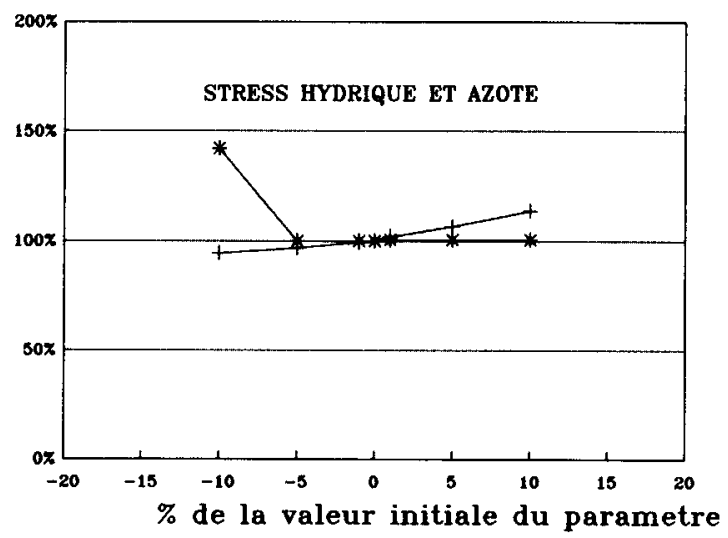

Fig 7. CERES-Maize. Test de sensibilité des parametres $P_{1}$ et $P_{5}$ en situation de stress hydrique et azoté. ( $\left.{ }^{*}\right) P_{1}$, somme de degrés-jour entre la levée et la fin de la phase juvénile; (+) $P_{5}$, somme de degrés-jour entre la floraison et la maturité physiologique. Valeurs initiales $(100 \%): P_{1}=125 ; P_{5}=720$.

intervalles de temps trop longs) ou même absente (date de la fin de la phase juvénile, biomasse racinaire, etc).

Le fonctionnement correct du modèle nécessite dans ces conditions, de déterminer les paramètres génétiques $P_{1}, P_{5}, G_{2}$ et $G_{3}$, et de modifier les équations qui calculent l'indice foliaire (LAI). Ces ajustements ne remettent pas en cause la structure du modèle. II serait donc abusif, à l'examen des seuls résultats obtenus en Alsace, de considérer la démarche des concepteurs de CERES-Maize comme inapplicable en Europe.

L'analyse de sensibilité montre que la réponse du modèle aux variations des paramètres n'est pas indépendante des conditions de simulation, par exemple en présence ou non d'un stress hydrique. La sélection des paramètres les plus sensibles en vue du calibrage doit donc reposer sur des analyses de sensibilité réalisées dans des conditions très variées.

Finalement, le modèle ne nous apparaît pas encore opérationnel dans les diverses conditions de culture du maïs rencontrées en Europe. II serait utile de tester spécialement la partie du modèle concernant la germination en raison des conditions d'implantation de la culture très différentes aux Etats-Unis et en Europe (printemps froid et humide). De plus, le travail réalisé sur une seule région ne permet pas de conclusions sur le bien-fondé des hypothèses majeures de CERES-Maize. La généralisation des résultats obtenus à l'échelle d'une région nécessite une évaluation multisite du modèle qui intègre des situations plus variées et plus nombreuses. Le travail réalisé en Alsace a néanmoins montré les principales contraintes d'une telle démarche : il s'agit d'une part de la difficulté d'obtenir un nombre suffisant de données fiables, complètes et répétées dans le temps, et d'autre part de choisir correctement les paramètres à calibrer et les critères de validation du modèle.

\section{RÉFÉRENCES}

Baanante CA, Godwin DC, Ritchie JT (1986) Economic evaluation of fertilizer strategies using the CERES models. Agron Abst $11 \mathrm{p}$

Berti WR, Karlen DL, Parsons JE (1986) Comparisons of measured and CERES-Maize-Predicted Corn Yields as affected by Soil Series. Agron Abst $11 \mathrm{p}$

Bloc D, Gay JP, Gouet JP (1983) Durée des phases végétative et reproductrice chez le maĩs. Influence de la température. Comm Colloque «Physiologie du maïs» Royan, 123-133

Gay JP, Bloc D (1983) L'élaboration du rendement. Détermination des composantes du rendement en grain. Comm Colloque "Physiologie du maïs" Royan, 181-192

Hodges T, Botner D, Sakamoto C, Hays Hang C (1987) Using the CERES-Maize model to estimate production for the US Cornbelt. Agric For Meteorol 40, 293-303

Jones CA, Kiniry JR (1986) CERES-Maize. A simulation model of maize growth and development. Texas A \& M Univ Press. College Stn. 194 p

Jones CA, Ritchie JT, Kiniry JR, Godwin DC, Otter SI (1984) The CERES Wheat and Maize Models. In: Proc Int Symp on Minimum Data Sets for Agrotechnol Trans. ICRISAT Center, India, Patancheru, 95100

Jorgensen SE (1988) Fundamental of ecological modelling. Developments in environmental modelling 9. Elsevier, Amsterdam, $391 \mathrm{p}$

Piper EL, Weiss A (1988) Evaluating CERES-Maize for response to Plant Population Reduction and Defoliation. Agron Abst $25 \mathrm{p}$

Wallach D, Goffinet B (1987) Mean square error of prediction in models for studying ecological and agronomic systems. Biometrics 43, 561-573

Whisler FD, Akock B, Baker DN, Fye RE, Hodges HF, Lambert JR, Emmon HE, McKinion M, Reddy VR (1986) Crop simulation models in agronomic systems. Adv Agron 40, 141-207 